\title{
CIENCIAMATRIA
}

Revista Interdisciplinaria de Humanidades, Educación, Ciencia y Tecnología

Año VIII. Vol. VIII. N¹4. Enero - Junio. 2022

Hecho el depósito de ley: pp201602FA4721

ISSN-L: 2542-3029; ISSN: 2610-802X

Universidad Nacional Experimental Francisco de Miranda (UNEFM). Santa Ana de Coro. Venezuela

Johnatan Amable Pilataxi-Chucad; Juan Carlos Arandia-Zambrano; Rously Eedyah Atencio-González

DOI $10.35381 / \mathrm{cm} . v 8 \mathrm{i} 14.639$

\section{La mediación en el derecho de familia y sus beneficios para las partes intervinientes}

\section{Mediation in family law and its benefits for the intervening parties}

\author{
Johnatan Amable Pilataxi-Chucad \\ dq.jonathanapc@uniandes.edu.ec \\ Universidad Regional Autónoma de los Andes, Quevedo \\ Ecuador \\ https://orcid.org/0000-0002-4211-5039 \\ Juan Carlos Arandia-Zambrano \\ uq.juanarandia@uniandes.edu.ec \\ Universidad Regional Autónoma de los Andes, Quevedo \\ Ecuador \\ https://orcid.org/0000-0003-1152-104X \\ Rously Eedyah Atencio-González \\ uq.rouslyatencio@uniandes.edu.ec \\ Universidad Regional Autónoma de los Andes, Quevedo \\ Ecuador \\ https://orcid.org/0000-0001-6845-1631
}

Recibido: 15 de septiembre 2021

Revisado: 10 de noviembre 2021

Aprobado: 15 de diciembre 2021

Publicado: 01 de enero 2022 


\title{
RESUMEN
}

El objetivo general de la investigación fue analizar la mediación en el derecho de familia y sus beneficios para las partes intervinientes. Se planteó a partir del enfoque cuantitativo, mediante la búsqueda, recolección y análisis crítico documental, referencial bibliográfico y estudios de investigación, desde un diseño bibliográfico de tipo documental, cuya pesquisa desde la deliberación crítica, se construyó el método de análisis del fenómeno, reflexionando leyes, normas, tratados internacionales. Como resultado se plantea que en el Ecuador la forma de someterse a mediación familiar se basa principalmente en la voluntariedad de las partes, ya sea de una solicitud directa o a través de derivación judicial. Se concluye que la aplicación de la mediación como forma alternativa de solución de conflictos en el derecho familiar, desarrollaría la capacidad de restructuración familiar, sin los efectos negativos que surgen del sometimiento a tribunales, que ayudaría a la aceptación de una nueva realidad social.

Descriptores: Mediación; derecho de la familia; solución de conflictos. (Tesauro UNESCO).

\begin{abstract}
The general objective of the research was to analyze mediation in family law and its benefits for the intervening parties. It was proposed from the quantitative approach, through the search, collection and critical documentary analysis, bibliographic reference and research studies, from a bibliographic design of documentary type, whose research from the critical deliberation, the method of analysis of the phenomenon was built, reflecting laws, regulations, international treaties. As a result, it is stated that in Ecuador the way of submitting to family mediation is based mainly on the voluntariness of the parties, either from a direct request or through judicial derivation. It is concluded that the application of mediation as an alternative form of conflict resolution in family law, would develop the capacity for family restructuring, without the negative effects arising from the submission to courts, which would help the acceptance of a new social reality.
\end{abstract}

Descriptors: Mediation; family law; conflict resolution. (UNESCO Thesaurus). 


\section{CIENCIAMATRIA}

Revista Interdisciplinaria de Humanidades, Educación, Ciencia y Tecnología

Año VIII. Vol. VIII. №14. Enero - Junio. 2022

Hecho el depósito de ley: pp201602FA4721

ISSN-L: 2542-3029; ISSN: 2610-802X

Universidad Nacional Experimental Francisco de Miranda (UNEFM). Santa Ana de Coro. Venezuela

Johnatan Amable Pilataxi-Chucad; Juan Carlos Arandia-Zambrano; Rously Eedyah Atencio-González

\section{INTRODUCCIÓN}

Las formas alternativas de solución de conflictos, han desarrollado un papel relevante en las reestructuraciones y modernizaciones que ha implementado el sector de justicia. Bajo esta modalidad, se enmarca, toda forma resolutiva de conflictos, que no tenga relación con la sentencia judicial, el desistimiento del conflicto o el uso de la fuerza. De esta forma se ha sostenido la ideología de que, existe la obligación por parte de los estados modernos y democráticos, de proveer sistemas de justicia que desarrollen distintas formas eficaces de solución de conflictos. (Stella Álvarez, 2014). La autora anteriormente citada establece que, la tutela de derechos que protege el estado constitucional del Ecuador, no se satisface únicamente a través del desarrollo de un poder judicial eficiente, pues este panorama, exige que el estado se apoye en la aplicación de mecanismos alternativos al judicial, en la resolución de controversias.

La figura jurídica de la mediación, se ha constituido como una institución reconocida por los diferentes instrumentos jurídicos nacionales, en los que encontramos a la (Constitución de la República del Ecuador, 2008), y la propia (Ley de Arbitraje y Mediación, 2006), entre otras leyes, por medio de las cuales, se desarrolla la facultad de las partes entramadas en un conflicto, a la concurrencia de centros de mediación, como medio alternativo de solución de conflictos, previo a la aplicación de la vía judicial, para lo cual se contara con el apoyo de un tercero imparcial, al cual se lo denomina como mediador. (Amaya López, 2020)

En el marco jurídico de la (Constitución de la República del Ecuador, 2008), la figura jurídica de la mediación, se reconoce en el artículo 190, en la cual se deja en constancia que, serán reconocidos el arbitraje, la mediación y demás métodos alternativos de solución de conflictos, dejando en constancia, que estos procedimientos se aplicarán sujetados a disposiciones legales, según la rama del derecho en las que, por su naturaleza, se pueda transigir. 


\section{CIENCIAMATRIA}

Revista Interdisciplinaria de Humanidades, Educación, Ciencia y Tecnología

Año VIII. Vol. VIII. №14. Enero - Junio. 2022

Hecho el depósito de ley: pp201602FA4721

ISSN-L: 2542-3029; ISSN: 2610-802X

Universidad Nacional Experimental Francisco de Miranda (UNEFM). Santa Ana de Coro. Venezuela

Johnatan Amable Pilataxi-Chucad; Juan Carlos Arandia-Zambrano; Rously Eedyah Atencio-González

La figura de la mediación, nace en consecuencia de diferentes factores, como son las problemáticas del sistema judicial, debido a la gran congestión que sufre este sistema, así como la insatisfacción que genera en la mayoría de casos la aplicación del sistema de justicia a través de resoluciones judiciales y el acatamiento de estas, de forma obligatoria. Es por ello que se debe fomentar un auge en la utilización de los métodos alternativos de resolución de controversias, en donde las partes intervinientes desarrollen un papel y participación fundamental, hasta el punto de poder encontrar un equilibrio de intereses que satisfaga a todas las partes que intervienen en esta metodología jurídica. (Folberg y Taylor, 1996)

Las definiciones y referencias que se han desarrollado sobre la figura de la mediación, pueden hacer pensar que esta figura desarrolla un proceso de orientación familiar, pero esto está totalmente alejado de la realidad. La gran diferencia que se desarrollan entre procesos de orientación familiar y la mediación es que la primera figura desarrolla solución a los problemas de pareja, fomentando una mejor comunicación, teniendo la finalidad de conseguir la no ruptura de la pareja, mientras que el proceso de mediación familiar, no desarrolla cuestiones de ruptura, puesto que para la mediación familiar, la ruptura de la pareja es un hecho, por lo que esta tiene la finalidad de redefinir esta nueva situación y buscar un ámbito beneficioso para ambas partes. Por todos los preceptos generados con anterioridad, es claro que la mediación se presenta como una forma que aporta muchos beneficios tanto a las partes como al sistema procesal, por ello radica su importancia de estudio, para poder analizar y desarrollar propuestas de mejoramiento de esta figura jurídica para que pueda generar aportes más significativos al sistema procesal ecuatoriano en relación al derecho de familia.

De acuerdo a los planteamientos formulados se plantea como objetivo general de la investigación analizar la mediación en el derecho de familia y sus beneficios para las partes intervinientes. 


\section{CIENCIAMATRIA}

Revista Interdisciplinaria de Humanidades, Educación, Ciencia y Tecnología

Año VIII. Vol. VIII. N¹4. Enero - Junio. 2022

Hecho el depósito de ley: pp201602FA4721

ISSN-L: 2542-3029; ISSN: 2610-802X

Universidad Nacional Experimental Francisco de Miranda (UNEFM). Santa Ana de Coro. Venezuela

Johnatan Amable Pilataxi-Chucad; Juan Carlos Arandia-Zambrano; Rously Eedyah Atencio-González

\section{METODOLOGÍA}

El trabajo investigativo se planteó a partir del enfoque cuantitativo, mediante la búsqueda, recolección y análisis crítico documental, referencial bibliográfico y estudios de investigación, desde la exégesis sobre la mediación en el derecho de familia y sus beneficios para las partes intervinientes en el contexto ecuatoriano, en este sentido, desde un diseño bibliográfico de tipo documental, cuya pesquisa desde la deliberación crítica, se construyó el método de análisis del fenómeno de estudio, reflexionando leyes, normas, acuerdos, tratados internacionales, lo que llevó a la producción de información relevante sobre el tema investigado.

\section{RESULTADOS}

Luego de la revisión documental-bibliográfica se presenta a continuación los resultados obtenidos:

En el Ecuador, en virtud de lo que establece el artículo 190, inciso primero de la actual Constitución Política de la República del Ecuador que reconoce el arbitraje, la mediación y otros procedimientos alternativos para la resolución de conflictos, con sujeción a la Ley, que someten las partes de mutuo acuerdo, sobre una materia transigible, de carácter extra judicial y definitivo, que ponga fin a un conflicto. En este sentido, en el Título II de la Ley de Arbitraje y Mediación (2006) en su Artículo. 43 desarrolla que: La mediación es un procedimiento de solución de conflictos.

Para obtener resultados claros, sobre los preceptos jurídicos que representan a la mediación como método alternativo de solución de conflictos, cabe destacar las siguientes afirmaciones desarrolladas por diferentes autores: 
CIENCIAMATRIA

Revista Interdisciplinaria de Humanidades, Educación, Ciencia y Tecnología

Año VIII. Vol. VIII. N¹4. Enero - Junio. 2022

Hecho el depósito de ley: pp201602FA4721

ISSN-L: 2542-3029; ISSN: 2610-802X

Universidad Nacional Experimental Francisco de Miranda (UNEFM). Santa Ana de Coro. Venezuela

Johnatan Amable Pilataxi-Chucad; Juan Carlos Arandia-Zambrano; Rously Eedyah Atencio-González

\section{Cuadro 1.}

Definiciones.

\begin{tabular}{|l|l|}
\hline \multicolumn{1}{|c|}{ Autores } & \multicolumn{1}{|c|}{ Afirmaciones } \\
\hline Pullupaxi Castro (2014). & $\begin{array}{l}\text { La mediación es un procedimiento de } \\
\text { solución de conflictos mediante el cual las } \\
\text { partes, asistidas por un tercero neutral } \\
\text { llamado mediador, procuran un acuerdo } \\
\text { voluntario, que verse sobre materia } \\
\text { transigible que ponga fin a un conflicto. }\end{array}$ \\
\hline Moore (1995). & $\begin{array}{l}\text { La Mediación es la intervención de una } \\
\text { disputa o negociación, de un tercero } \\
\text { aceptable, imparcial y neutral que carece de } \\
\text { un poder autorizado de decisión para ayudar } \\
\text { a las partes en disputa a alcanzar } \\
\text { voluntariamente su propio arreglo } \\
\text { mutuamente aceptable. La Mediación es } \\
\text { esencialmente la negociación que incluye a } \\
\text { un tercero que conoce los procedimientos } \\
\text { eficaces de negociación y puede ayudar a la } \\
\text { gente en conflicto a coordinar sus } \\
\text { actividades y ser más eficaces en su pugna. }\end{array}$ \\
\hline $\begin{array}{l}\text { La mediación es la intervención de una } \\
\text { tercera parte, competente e imparcial, en }\end{array}$ \\
una disputa, con el propósito de ayudar a las \\
partes a resolver sus diferencias y a mejorar \\
sus relaciones en el futuro, proveyéndoles \\
de un ambiente seguro.
\end{tabular}

Elaboración: Los autores. 


\section{CIENCIAMATRIA}

Revista Interdisciplinaria de Humanidades, Educación, Ciencia y Tecnología

Año VIII. Vol. VIII. №14. Enero - Junio. 2022

Hecho el depósito de ley: pp201602FA4721

ISSN-L: 2542-3029; ISSN: 2610-802X

Universidad Nacional Experimental Francisco de Miranda (UNEFM). Santa Ana de Coro. Venezuela

Johnatan Amable Pilataxi-Chucad; Juan Carlos Arandia-Zambrano; Rously Eedyah Atencio-González

Para el desarrollo de las ventajas y desventajas que presenta esta figura jurídica, es necesario mencionar a los autores Herrera Garcés y Camalle Cumbal (2017), el cual establece que la mediación tiene varias ventajas tales como:

a) Los costos que demanda un proceso de mediación son significativamente menores que los demandados por otros medios judiciales de solución de conflictos.

b) Soluciones creativas. El proceso de mediación permite crear y a la vez explorar soluciones creativas, buscando la mejor fórmula para llegar a un acuerdo que beneficie a las partes poniendo fin al conflicto, circunstancia que obedece a los intereses reales de todas las partes involucradas.

c) En esta parte el mediador tiene que aplicar un sinnúmero de técnicas con habilidad y destreza que le permitan ayudar a las partes y por ende buscar la solución que se adapte a las necesidades de las mismas.

d) Control sobre el resultado. En un proceso de mediación son las partes quienes tienen y mantiene el control sobre el resultado del conflicto y por tanto decidir un acuerdo total parcial, a diferencia de lo que acontece en los procedimientos en los cuales la decisión para la resolución de un conflicto está sometida a un tercero (juez).

La figura de la mediación también presenta desventajas, más sin embargo si las analizamos frente a las ventajas, estas desventajas son menores:

1. Inasistencia. - Al ser la Mediación un proceso voluntario, hace que sea confundido con un trámite sin valor, donde incluso muchas de las partes convocadas a mediar, ni si quiera se presentan a las audiencias de Mediación, con lo cual resta efectividad a la misma.

2. Falta de capacitación de los mediadores. - Es importante tener en cuenta que generalmente los asuntos sometidos a Mediación son hechos familiares, de trabajo, niñez, etc. es decir generalmente entre las partes no solamente existen un conflicto de intereses, o económicos, sino conflictos sentimentales, lo cual adhiere 


\section{CIENCIAMATRIA}

Revista Interdisciplinaria de Humanidades, Educación, Ciencia y Tecnología

Año VIII. Vol. VIII. №14. Enero - Junio. 2022

Hecho el depósito de ley: pp201602FA4721

ISSN-L: 2542-3029; ISSN: 2610-802X

Universidad Nacional Experimental Francisco de Miranda (UNEFM). Santa Ana de Coro. Venezuela

Johnatan Amable Pilataxi-Chucad; Juan Carlos Arandia-Zambrano; Rously Eedyah Atencio-González

un factor más de solución en el proceso de Mediación, he ahí la importancia de contar con un mediador completamente capacitado, que sin llegar a entrometerse o forzar a las partes a un acuerdo, sea lo suficientemente inteligente y conciliador, que permita ir superando ciertos problemas personales, que de pronto siempre van a seguir ahí, pero que no implica que se pueda solucionar los conflictos que llevó a las partes a media.

3. Falta de presión por parte de los mediadores. - Este punto es bastante álgido y debe ser entendido, con falta de presión no significa que el mediador no cerrará la audiencia sin conseguir a toda costa un arreglo entre las partes, pues esto iría contra la naturaleza de la Mediación, es decir contra su carácter de voluntaria, de cesión de posturas y de conveniencia mutua.

4. Escasez de Tiempo.- Hay procesos de Mediación que pueden ser abordados en cuestión de minutos y máximo horas, pero hay otros procesos de Mediación que requieren mucho más que un par de minutos para encontrar el camino de solución de conflictos, sino realmente una o varias audiencias a fin de ser escuchados, ir entendiendo el origen y sentido de la Mediación, es por eso que no cabe un proceso de Mediación limitado a tiempos, el sistema debe ser más flexible y sensato, y dar la oportunidad a los centros de Mediación de manejar los casos dentro de un margen de tiempo racional, y a razón de los temas que se discuten, pues unos llevan implícita mayor complejidad, incluso emocional que otro.

\section{La mediación en el derecho de familia}

La mediación familiar consiste en el uso de este método alternativo de resolución de conflictos en el ámbito del Derecho de Familia. El objetivo es llegar a la solución integral de un conflicto entre partes (pueden ser dos o más personas) evitando llegar a la instancia judicial. 


\section{CIENCIAMATRIA}

Revista Interdisciplinaria de Humanidades, Educación, Ciencia y Tecnología

Año VIII. Vol. VIII. №14. Enero - Junio. 2022

Hecho el depósito de ley: pp201602FA4721

ISSN-L: 2542-3029; ISSN: 2610-802X

Universidad Nacional Experimental Francisco de Miranda (UNEFM). Santa Ana de Coro. Venezuela

Johnatan Amable Pilataxi-Chucad; Juan Carlos Arandia-Zambrano; Rously Eedyah Atencio-González

Hay múltiples formas de poner en marcha este tipo de procesos, y distintos países en todo el mundo la aplican de diferente modo, persiguiendo siempre la misma meta: alcanzar un acuerdo duradero y justo, fruto del compromiso y del entendimiento entre las partes.

En el Ecuador la forma de someterse a mediación familiar se basa principalmente en la voluntariedad de las partes, al sometimiento de la misma, a través ya sea de una solicitud directa presentada por los usuarios de forma individual o a través de derivación judicial, cuando es un juzgador que a petición de parte o de oficio remite al centro de mediación de la función judicial una causa, hechos que generan una utilización mínima de este tipo de procedimientos, puesto que, en materia de familia, las reestructuraciones familiares que se producen, impiden en la mayoría de casos un buen entendimiento en primera instancia de las parejas en proceso de separación.

\section{DISCUSIÓN}

La mediación jurídica es un mecanismo alternativo de solución de conflictos por el cual las partes, asistidas por un tercero neutral llamado mediador, procuran un acuerdo voluntario, que verse sobre materia transigible, de carácter extrajudicial y definitivo, que ponga fin al conflicto, este proceso está reconocido tanto por la constitución como por mecanismos de carácter internacional como lo es la carta constitutiva de las Naciones Unidas, el cual establece a la mediación como medio para el arreglo pacífico de las controversias.

En este plano y ante esta incógnita, es notorio, que la mediación jurídica, dentro del derecho de familia se torna como una forma real y alternativa de solución de conflictos, puesto que posee características y medios, mediante los cuales se permite desarrollar los intereses y disputas del derecho de familia con mayor rapidez y eficacia, que, a través del procedimiento judicial, en el cual este tipo de controversias, se ven ralentizadas por las formalidades y plazos judiciales, así como por el congestionamiento de causas que 


\begin{abstract}
CIENCIAMATRIA
Revista Interdisciplinaria de Humanidades, Educación, Ciencia y Tecnología

Año VIII. Vol. VIII. N¹4. Enero - Junio. 2022

Hecho el depósito de ley: pp201602FA4721

ISSN-L: 2542-3029; ISSN: 2610-802X

Universidad Nacional Experimental Francisco de Miranda (UNEFM). Santa Ana de Coro. Venezuela

Johnatan Amable Pilataxi-Chucad; Juan Carlos Arandia-Zambrano; Rously Eedyah Atencio-González
\end{abstract}

existe en el sistema procesal civil ecuatoriano en la actualidad.

\title{
CONCLUSIÓN
}

Se llega a la conclusión de que, la mediación es un método alternativo de resolución de conflictos, el cual está reconocido por la constitución del ecuador como por instrumentos internacionales, el cual tiene la finalidad de generar acuerdos beneficiosos para las partes intervinientes en conjunto con un tercero neutral al cual se lo denomina como mediador, encargado de mediante la aplicación de técnicas, de desarrollar un ambiente en el que los intervinientes puedan generar acuerdos donde ambas partes satisfagan sus intereses, hecho que genera bienestar en todas las partes, al contrario que la resolución judicial, donde solo una de las partes, materializa sus intereses.

Los métodos alternativos de solución de conflictos, están correctamente regulados y reconocidos por el estado ecuatoriano, pero la voluntad de sometimiento a estos procesos que prima en el desarrollo de estos conflictos, genera un efecto de utilización de estas herramientas casi inexistente, por ello es clara la necesidad de desarrollar una utilización preferente de este mecanismo alternativo de solución de conflictos en el derecho de familia, para maximizar su efectividad y contribuir al descongestionamiento del sistema procesal ecuatoriano.

La aplicación de la mediación como forma alternativa de solución de conflictos en el derecho familiar, desarrollaría la capacidad de restructuración familiar, sin los efectos negativos que surgen del sometimiento a tribunales, desarrollando un estado de tolerancia para todas las partes intervinientes que ayudaría a la aceptación de una nueva realidad social.

\section{FINANCIAMIENTO}

No monetario. 
CIENCIAMATRIA

Revista Interdisciplinaria de Humanidades, Educación, Ciencia y Tecnología

Año VIII. Vol. VIII. N¹4. Enero - Junio. 2022

Hecho el depósito de ley: pp201602FA4721

ISSN-L: 2542-3029; ISSN: 2610-802X

Universidad Nacional Experimental Francisco de Miranda (UNEFM). Santa Ana de Coro. Venezuela

Johnatan Amable Pilataxi-Chucad; Juan Carlos Arandia-Zambrano; Rously Eedyah Atencio-González

\section{AGRADECIMIENTO}

A la Universidad Regional Autónoma de los Andes, Quevedo, por motivar el desarrollo de la Investigación.

\section{REFERENCIAS CONSULTADAS}

Amaya López, C., y Juanes Giraud, B. (2020). Descongestión del sistema judicial en Ecuador. Método alternativo de solución de conflictos en la mediación en primera instancia en materia laboral. [Decongestion of the judicial system in Ecuador. Alternative method of conflict resolution in mediation in the first instance in labor matters] Revista Universidad y Sociedad, 12(5), 518-524. Recuperado de: https://n9.cl/stp4b

Asamblea Nacional Constituyente de la República del Ecuador, (2008). Constitución de la República del Ecuador. [Constitution of the Republic of Ecuador].Montecristi. Registro Oficial 449 de 20-oct-2008. Recuperado de https://n9.cl/sia

Congreso Nacional (2006) Ley de Arbitraje y Mediación. Codificación de la ley de arbitraje y mediación. [Law on Arbitration and Mediation. Codification of the law of arbitration and mediation] Ley No. 2006-014. Registro Oficial No. 17 DE 14-DIC-2006. Recuperado de: https://n9.cl/b9t4

Folberg, J., y Taylor, A. (1996). Mediación: Resolución de conflictos sin litigio. [Mediation: Dispute resolution without litigation] México D.F.: LIMUSA. Noriega Editores. Recuperado de: https://n9.cl/t1loev

Herrera Garcés, S., y Camalle Cumbal, M. (2017) La mediación como instrumento efectivo para la solución de conflictos individuales del trabajo en la Legislación Ecuatoriana año 2015. [Mediation as an effective instrument for the resolution of individual labor disputes in Ecuadorian Legislation 2015] Tesis de pregrado. Universidad Central del Ecuador. Recuperado de: https://n9.cl/sgzfk

Martínez-Pecino,R., Munduate, L., y Medina, F.(2008) La gestión de conflictos organizacionales por medios extrajudiciales [organizational conflict management by out-of-court means] Papeles del Psicólogo,29(1),41-48. Recuperado de: https://n9.cl/b43xq 


\section{CIENCIAMATRIA}

Revista Interdisciplinaria de Humanidades, Educación, Ciencia y Tecnología

Año VIII. Vol. VIII. N¹4. Enero - Junio. 2022

Hecho el depósito de ley: pp201602FA4721

ISSN-L: 2542-3029; ISSN: 2610-802X

Universidad Nacional Experimental Francisco de Miranda (UNEFM). Santa Ana de Coro. Venezuela

Johnatan Amable Pilataxi-Chucad; Juan Carlos Arandia-Zambrano; Rously Eedyah Atencio-González

Moore, C. W. (1995). El proceso de mediación, métodos prácticos para la solución de conflictos. [The mediation process, practical methods for conflict resolution] Granica S.A. Buenos Aires. Argentina.

Pullupaxi Castro, F. (2014) La mediación y los conflictos individuales de trabajo en la legislación ecuatoriana.[ Mediation and individual labour disputes in Ecuadorian law] Tesis de pregrado. Universidad Técnica de Ambato. Recuperado de: https://n9.cl/n9e19

Stella Álvarez, G. (2014). Los métodos alternativos de solución de conflictos en los procesos judiciales: experiencias argentinas. [Alternative methods of conflict resolution in judicial processes: Argentine experiences] Departamento de Derecho Internacional OEA, Buenos Aires, Argentina. Recuperado de: https://n9.cl/o5jwn

Touzard, H. (1980). La mediación y la solución de los conflictos. [Mediation and conflict resolution]. Barcelona. España. Herder.

C2022 por los autores. Este artículo es de acceso abierto y distribuido según los términos y condiciones de la licencia Creative Commons Atribución-NoComercial-Compartirlgual 4.0 Internacional (CC BY-NC-SA 4.0)

(https://creativecommons.org/licenses/by-nc-sa/4.0/). 